PROCEEDINGS OF THE

AMERICAN MATHEMATICAL SOCIETY

Volume 137, Number 3, March 2009, Pages 1039-1049

S 0002-9939(08)09659-7

Article electronically published on September 25, 2008

\title{
A TRACE FORMULA AND SCHMINCKE INEQUALITY ON THE HALF-LINE
}

\author{
AMIN BOUMENIR AND VU KIM TUAN \\ (Communicated by Walter Craig)
}

\begin{abstract}
In this paper we derive a trace formula for the Schrödinger operator on the half-line. As a consequence we obtain a Schmincke type inequality with sharp constant. The main tool in our investigation is the inverse spectral Gelfand-Levitan theory, which allows us to compare two Schrödinger operators whose spectra differ by few eigenvalues.
\end{abstract}

\section{Introduction AND MAin RESUlts}

Lieb and Thirring 11 have shown that the sum of the moments of the negative eigenvalues $-\lambda_{1} \leq-\lambda_{2} \leq \cdots \leq 0$ (if any) of the Schrödinger operator $-\Delta-V$ on $L^{2}\left(\mathbb{R}^{d}\right)$ is bounded by

$$
\sum \lambda_{i}^{\gamma} \leq L_{\gamma, d} \int_{\mathbb{R}^{d}}\left(V_{-}(x)\right)^{\gamma+d / 2} d x
$$

where $V_{-}(x):=\max \{V(x), 0\}$. One of the challenges is to find the smallest possible constant $L_{\gamma, d}$, known as the sharp constant in (1.1). For the sake of simplicity we shall restrict ourselves to eigenvalue inequalities in the case $d=1$.

It is well known that if $d=1$, then (1.1) cannot hold for $\gamma<1 / 2$. For the limit case $\gamma=1 / 2$, Hundertmark, Lieb, and Thomas [5] have shown that if $V_{-} \in L^{1}(\mathbb{R})$, then

$$
\sum \sqrt{\lambda_{i}} \leq L_{1 / 2,1} \int_{-\infty}^{\infty} V_{-}(x) d x
$$

where $L_{1 / 2,1}=\frac{1}{2}$ is a sharp constant. The main tool used in deriving (1.2) is the Birman-Schwinger principle which relates negative eigenvalues of a Schrödinger operator with the eigenvalues of a certain integral operator.

If $V$ is continuous, $V(x) \rightarrow 0$ as $|x| \rightarrow \infty$ and $\int_{\mathbb{R}} V(x) d x$ exists (possibly conditionally), Schmincke [13] uses the commutation method to obtain the lower bound

Received by the editors March 31, 2008.

2000 Mathematics Subject Classification. Primary 34L15, 34A55.

Key words and phrases. Trace formula, negative eigenvalues, Schmincke inequality, inverse spectral theory.

(C)2008 American Mathematical Society 
for the sum of the negative eigenvalues

$$
\frac{1}{4} \int_{-\infty}^{\infty} V(x) d x \leq \sum \sqrt{\lambda_{i}}
$$

and here $\frac{1}{4}$ is a sharp constant.

If we also assume that $(1+|x|) V(x) \in L^{1}(\mathbb{R})$, then Schmincke's inequality (1.3) follows at once from the Faddeev-Zakharov trace formula [15]

$$
\int_{-\infty}^{\infty} V(x) d x=4 \sum \sqrt{\lambda_{i}}+\frac{1}{\pi} \int_{-\infty}^{\infty} \ln \left(1-|R(k)|^{2}\right) d k
$$

since the reflection coefficient of the operator $H$ satisfies $R(k) \in[0,1]$.

A well-known fact in the spectral theory of operators is that negative eigenvalues depend on the self-adjoint extensions, 12. Also if a Lieb-Thirring inequality holds, it must do so for all isospectral operators, since it does not depend on the energy of the bound states. Thus to shed some light on these hidden connections we study the sum of the negative eigenvalues of a Schrödinger operator on the half-line, with exponent $\gamma=1 / 2$, and under the sole condition that $q \in L^{1}(0, \infty)$. Thus we consider the one-dimensional self-adjoint Schrödinger operator on the half-line defined by

$$
\left\{\begin{array}{l}
H(y):=-y^{\prime \prime}(x, \lambda)-q(x) y(x, \lambda)=-\lambda y(x, \lambda), \quad x \in[0, \infty), \\
y^{\prime}(0, \lambda)-h y(0, \lambda)=0, \text { where } h, q(x) \in \mathbb{R} .
\end{array}\right.
$$

The spectrum of $H$ then is continuous on the positive half-line $[0, \infty)$, bounded and discrete on the negative half-line. From now on we assume that $H$ has only finitely many negative eigenvalues (which is the case when $(1+x) q(x) \in L^{1}(0, \infty)$. Denote these negative eigenvalues by $-\lambda_{1},-\lambda_{2}, \cdots,-\lambda_{N}(N \geq 0)$. We use the notation $q_{N}$ interchangeably with $q$ to specify that $H$ then has exactly $N$ negative eigenvalues. Recall a known result by Weidl [14] who showed that if $q(x) \geq 0, q \in L^{1}(0, \infty)$, and $h=0$, then the Lieb-Thirring inequality (1.2) holds with $L_{\frac{1}{2}, 1}<1.005$.

Let $\alpha_{j}=1 / \int_{0}^{\infty}\left|y\left(x,-\lambda_{i}\right)\right|^{2} d x$ be the norming constant, which represents the jump size of the spectral function [10] at $-\lambda_{j}$. The main result of this paper is the trace formula

$$
\int_{0}^{\infty} q_{N}(x) d x=\int_{0}^{\infty} q_{0}(x) d x-2 \sum_{j=1}^{N} \alpha_{j}+4 \sum_{j=1}^{N} \sqrt{\lambda_{j}},
$$

where $q_{0}$ is obtained from $q_{N}$ by removing all $N$ negative eigenvalues. The appearance of the norming constants $\alpha_{j}$ distinguishes (1.6) from the Faddeev-Zakharov trace formula (1.4) and brings out a new relation between isospectral operators. We then prove that if $q_{0}$ generates no negative eigenvalues, then the estimate

$$
\int_{0}^{\infty} q_{0}(x) d x \leq h_{0}
$$

holds, which yields the Schmincke inequality for the half-line

$$
\frac{1}{4} \int_{0}^{\infty} q(x) d x-\frac{h}{4}<\sum \sqrt{\lambda_{j}} .
$$

Observe that inequality (1.8) is different from (1.3) by the boundary term $-\frac{h}{4}$ and that inequality (1.8) is proved here without the usual restrictions on $q$. For example in [13] it is assumed that $q$ is continuous and $q \rightarrow 0$ as $x \rightarrow \infty$, while in [14] it is 
required that $(1+x) q(x) \in L^{1}(0, \infty)$ for the Faddeev-Zakharov trace formula (1.4) to hold.

The main tool in deriving (1.6) is the inverse spectral Gelfand-Levitan theory, [10], which allows us to construct a potential $q$ explicitly in terms of the negative eigenvalues. The difficulty lies in the fact that the potential $q$ also depends on the positive spectrum, and the proof of inequality (1.7) without any smoothness condition imposed on $q_{0}$ is very technical; see Lemma 8 in 13 . for a similar equality for a continuous potential $q_{0}$.

Note that an alternative to the Gelfand-Levitan method is the double commutation method, 7]. In [6], Gesztesy and Teschl used it to insert finitely many eigenvalues in spectra of more general singular Sturm-Liouville operators generated by

$$
l(y)=\frac{1}{k(x)}\left(-\left(p(x) y^{\prime}(x)\right)^{\prime}+q(x) y(x)\right), \text { where }-\infty \leq a \leq x \leq b \leq \infty .
$$

Integrating equation (4.5) in [6], with suitable asymptotics should yield a similar relation to (1.6).

Observe that (1.6) has the advantage of measuring the gap of the inequality, which gives new insight on the sharp constant in (1.3). We also derive a second inequality, which involves the product of eigenvalues

$$
\int_{0}^{\infty} x q_{N}(x) d x=\int_{0}^{\infty} x q_{0}(x) d x+\ln \left(\frac{4 \prod_{j=1}^{N} \lambda_{j}}{\prod_{j=1}^{N} \alpha_{j}^{2}}\right),
$$

in case $(1+x) q(x) \in L^{1}(0, \infty)$.

\section{A traCe FORMUla}

Without loss of generality, let $H_{N}$ denote the self-adjoint Schrödinger operator on the half-line (1.5) whose spectrum has exactly $N$ negative eigenvalues, say $0>$ $-\lambda_{1} \geq-\lambda_{2} \geq \cdots \geq-\lambda_{N}$. Denote its potential by $q_{N} \in L^{1}(0, \infty)$ and its spectral function by $\rho_{N}(\lambda)=\rho(\lambda)$. The jump of $\rho_{N}$ at $-\lambda_{j}$ is denoted by $\alpha_{j}$ and by $\rho_{k}(\lambda)$, $k=0,1, \cdots, N-1$; we denote the spectral functions obtained from $\rho_{N}(\lambda)$ by removing the jumps at $-\lambda_{k+1}, \cdots,-\lambda_{N}$, i.e.

$$
\rho_{k}(\lambda)=\rho_{N}(\lambda)-\sum_{j=k+1}^{N} \alpha_{j} \mathbf{H}\left(\lambda+\lambda_{j}\right),
$$

where $\mathbf{H}$ is the usual Heaviside function. The last spectral function $\rho_{0}$ is constant for $\lambda \leq 0$, since we have no negative eigenvalue. Let $H_{k}$, where $k=0, \ldots, N$, denote the family of Schrödinger operators on the half-line, having $\rho_{k}$ as the spectral functions

$$
\left\{\begin{array}{l}
H_{k}\left(y_{k}\right):=-y_{k}^{\prime \prime}(x, \lambda)-q_{k}(x) y_{k}(x, \lambda)=-\lambda y_{k}(x, \lambda), \quad x \in[0, \infty), \\
y_{k}^{\prime}(0, \lambda)-h_{k} y_{k}(0, \lambda)=0 .
\end{array}\right.
$$

The potentials $q_{k}$ are locally integrable, $q_{k} \in L^{1, l o c}(0, \infty)$, and all eigensolutions, $y_{k}(., \lambda)$, are normalized by $y_{k}(0, \lambda)=1$.

The Gelfand-Levitan theory [10] guarantees the existence and uniqueness of operators $H_{k}$ when appropriate $\rho_{k}$ are given. In fact adding one eigenvalue to a spectrum is a simple operation, [10, and the relation between $q_{k}$ and $q_{k+1}$ is

$$
q_{k+1}(x)=q_{k}(x)+2 \alpha_{k+1} \frac{d}{d x} \frac{y_{k}^{2}\left(x,-\lambda_{k+1}\right)}{1+\alpha_{k+1} \int_{0}^{x} y_{k}^{2}\left(s,-\lambda_{k+1}\right) d s} .
$$


Here the appearance of the positive sign before the differential operator $\frac{d}{d x}$ is due to the fact that we are dealing with $-q_{k}$ instead of the usual $+q_{k}$ in (2.1).

To remove one eigenvalue at a time from the spectrum, we simply reverse the method in [10]. In other words, we will find a formula to go from $q_{k+1}$ to $q_{k}$. To this end, denote by

$$
\begin{aligned}
F_{k}(x, t) & =\int_{-\infty}^{\infty} y_{k+1}(x, \lambda) y_{k+1}(t, \lambda) d\left(\rho_{k}(\lambda)-\rho_{k+1}(\lambda)\right) \\
& =-\alpha_{k+1} y_{k+1}\left(x,-\lambda_{k+1}\right) y_{k+1}\left(t,-\lambda_{k+1}\right)
\end{aligned}
$$

and let $K_{k}(x, t)$ be the solution of the well-known linear Fredholm integral equation

$$
F_{k}(x, t)+K_{k}(x, t)+\int_{0}^{x} K_{k}(x, s) F_{k}(s, t) d s=0 \quad \text { for } \quad 0 \leq t \leq x .
$$

Since the kernel $F_{k}$ is a degenerate kernel, the solution is simply of the form $K_{k}(x, t)=\alpha_{k+1} \beta(x) y_{k+1}\left(t,-\lambda_{k+1}\right)$, where

$$
\beta(x)\left(1-\alpha_{k+1} \int_{0}^{x} y_{k+1}^{2}\left(s,-\lambda_{k+1}\right) d s\right)=y_{k+1}\left(x,-\lambda_{k+1}\right),
$$

i.e.

and

$$
K_{k}(x, t)=\frac{\alpha_{k+1} y_{k+1}\left(x,-\lambda_{k+1}\right) y_{k+1}\left(t,-\lambda_{k+1}\right)}{1-\alpha_{k+1} \int_{0}^{x} y_{k+1}^{2}\left(s,-\lambda_{k+1}\right) d s}
$$

$$
K_{k}(0,0)=\alpha_{k+1} y_{k+1}\left(0,-\lambda_{k+1}\right) y_{k+1}\left(0,-\lambda_{k+1}\right)=\alpha_{k+1} .
$$

Thus we can determine a new potential $q_{k}$ from $q_{k+1}$ by the following formula [10]:

$$
q_{k}(x)=q_{k+1}(x)-2 \frac{d}{d x} K_{k}(x, x) .
$$

Integrating (2.4) from 0 to $x$ yields

$$
\int_{0}^{x} q_{k+1}(\eta) d \eta=\int_{0}^{x} q_{k}(\eta) d \eta+2 \alpha_{k+1}\left(\frac{y_{k+1}^{2}\left(x,-\lambda_{k+1}\right)}{1-\alpha_{k+1} \int_{0}^{x} y_{k+1}^{2}\left(s,-\lambda_{k+1}\right) d s}-1\right) .
$$

An obvious way to bring out eigenvalues is to take the limit as $x \rightarrow \infty$. To this end we need the following lemma.

Lemma 1. Consider the operator $H$ in (1.5) where $q \in L^{1}(0, \infty)$. If $-\lambda_{*} \in \sigma$ is a negative eigenvalue, then $y\left(x,-\lambda_{*}\right) \approx a\left(\lambda^{*}\right) \exp \left(-x \sqrt{\lambda_{*}}\right)$, and $y^{\prime}\left(x,-\lambda_{*}\right) \approx$ $-\sqrt{\lambda^{*}} a\left(\lambda^{*}\right) \exp \left(-x \sqrt{\lambda_{*}}\right)$, as $x \rightarrow \infty$. and $a\left(\lambda^{*}\right) \neq 0$.

Proof. Since $q \in L^{1}(0, \infty)$, for large $x \rightarrow \infty$, we have $y(x,-\lambda) \approx a(\lambda) \exp (-x \sqrt{\lambda})$ $+b(\lambda) \exp (x \sqrt{\lambda})$, [12, Thm. 8, Section 22]. Since $-\lambda^{*} \in \sigma$, then $y\left(.,-\lambda^{*}\right) \in$ $L^{2}(0, \infty)$ and so $b\left(\lambda^{*}\right)=0$ but $a\left(\lambda^{*}\right) \neq 0$.

As $-\lambda_{k+1}$ is an eigenvalue of $H_{k+1}$, and since

$$
\int_{0}^{\infty} y_{k+1}^{2}\left(s,-\lambda_{k+1}\right) d s=\frac{1}{\alpha_{k+1}},
$$

by Lemma 1 and l'Hospital's rule we have

$$
\lim _{x \rightarrow \infty} \frac{2 \alpha_{k+1} y_{k+1}^{2}\left(x,-\lambda_{k+1}\right)}{1-\alpha_{k+1} \int_{0}^{x} y_{k+1}^{2}\left(s,-\lambda_{k+1}\right) d s}=-\lim _{x \rightarrow \infty} \frac{4 y_{k+1}^{\prime}\left(x,-\lambda_{k+1}\right)}{y_{k+1}\left(x,-\lambda_{k+1}\right)}=4 \sqrt{\lambda_{k+1}}
$$


The existence of the limit of the right-hand side of (2.5), as $x \rightarrow \infty$, then yields

$$
\int_{0}^{\infty} q_{k+1}(\eta) d \eta=\int_{0}^{\infty} q_{k}(\eta) d \eta+4 \sqrt{\lambda_{k+1}}-2 \alpha_{k+1} .
$$

Next we need to verify that if $q_{k+1}$ is integrable, then the newly constructed $q_{k}$ is also integrable.

Lemma 2. Let $q_{k+1} \in L^{1}(0, \infty)$ and $q_{k}$ be defined by (2.4). Then $q_{k} \in L^{1}(0, \infty)$.

Proof. By (2.4) we only need to show that $\frac{d}{d x} K_{k}(x, x) \in L^{1}(0, \infty)$. From the asymptotics of $y_{k+1}\left(x,-\lambda_{k+1}\right)$ at infinity (Lemma 1) it is not difficult to see that $\frac{d}{d x} K_{k}(x, x)<0$ for large $x$, say $x>M$. Then

$$
\int_{M}^{\infty}\left|\frac{d}{d x} K_{k}(x, x)\right| d x=-\int_{M}^{\infty} \frac{d}{d x} K_{k}(x, x) d x=K_{k}(M)+2 \lambda_{k+1}<\infty
$$

and so $\frac{d}{d x} K_{k}(x, x) \in L^{1}(0, \infty)$, and therefore $q_{k} \in L^{1}(0, \infty)$.

We now can repeat the same procedure, which yields after finite steps,

$$
\int_{0}^{\infty} q_{N}(x) d x=\int_{0}^{\infty} q_{0}(x) d x-2 \sum_{j=1}^{N} \alpha_{j}+4 \sum_{j=1}^{N} \sqrt{\lambda_{j}} .
$$

Thus we have proved:

Theorem 1 (trace formula). Assume that $q_{N} \in L^{1}(0, \infty)$ has only finitely many negative eigenvalues, and $q_{0}$ is obtained from $q_{N}$ by removing all negative eigenvalues of $H_{N}$. Then $q_{0} \in L^{1}(0, \infty)$, and identity (2.8) holds.

Observe that the value of $h_{0}$, which appears in the boundary condition at $x=0$, can be computed explicitly in terms of the norming constants $\alpha_{k}$. Clearly since $y_{k+1}(0, \lambda)=1$, then

$$
h_{k}=y_{k}^{\prime}(0, \lambda)=y_{k+1}^{\prime}(0, \lambda)+K_{k}(0,0)=h_{k+1}+\alpha_{k+1} .
$$

Tracking down the sequence of constructed eigensolutions we have been using, with the notation $\left(\begin{array}{l}y_{k}(0, \lambda) \\ y_{k}^{\prime}(0, \lambda)\end{array}\right)$, we have

$$
\left(\begin{array}{c}
1 \\
h_{N}
\end{array}\right) \Rightarrow\left(\begin{array}{c}
1 \\
h_{N}+\alpha_{N}
\end{array}\right) \Rightarrow\left(\begin{array}{c}
1 \\
h_{N}+\alpha_{N}+\alpha_{N-1}
\end{array}\right) \Rightarrow \cdots \Rightarrow\left(\begin{array}{c}
1 \\
h_{N}+\sum_{j=1}^{N} \alpha_{j}
\end{array}\right) .
$$

Observe that we could have started with any $h_{N}$ and still ended with $h_{0}=h_{N}+$ $\sum_{j=1}^{N} \alpha_{j}>h_{N}$.

Corollary 1. Assume that the conditions of Theorem 1 hold. Then $h_{0}>h_{N}$, and

$$
\begin{aligned}
\int_{0}^{\infty} q_{N}(x) d x & =\int_{0}^{\infty} q_{0}(x) d x+2\left(h_{N}-h_{0}\right)+4 \sum_{j=1}^{N} \sqrt{\lambda_{j}}, \\
\int_{0}^{\infty} q_{N}(x) d x-h_{N} & =\int_{0}^{\infty} q_{0}(x) d x-h_{0}-\sum_{j=1}^{N} \alpha_{j}+4 \sum_{j=1}^{N} \sqrt{\lambda_{j}} .
\end{aligned}
$$

Since we do not have a restriction on the sign of $q$ and the $\lambda_{j}$ are arbitrary,

Corollary 2. We have $h_{0}-h_{N}=2 \sum_{j=1}^{N} \sqrt{\lambda_{j}} \quad$ if and only if $\int_{0}^{\infty} q_{N}(x) d x=$ $\int_{0}^{\infty} q_{0}(x) d x$. 


\section{The COntinuous SPECTRUm CASE}

In order to compare the sum of moments of the negative eigenvalues with $\int_{0}^{\infty} q_{N}(x) d x-h_{N}$, from (2.9) we only need to estimate $\int_{0}^{\infty} q_{0}(x) d x-h_{0}$, which is determined by the spectral function over $[0, \infty)$. Recall that the eigensolution $y_{0}(x, \lambda)$ of $H_{0}$ satisfies the transmutation equation [10]

$$
\cos (x \sqrt{\lambda})=y_{0}(x, \lambda)+\int_{0}^{x} G_{0}(x, t) y_{0}(t, \lambda) d t,
$$

where

$$
G_{0}(x, x)=\frac{1}{2} \int_{0}^{x} q_{0}(\eta) d \eta-h_{0} .
$$

On the other hand, the kernel function $G_{0}(x, t)$ satisfies the nonlinear integral equation [3, 10]

$$
F(x, t)=G_{0}(t, x)+G_{0}(x, t)+\int_{0}^{x} G_{0}(x, \eta) G_{0}(t, \eta) d \eta,
$$

where

$$
F(x, t)=\int_{0}^{\infty} \cos (x \sqrt{\lambda}) \cos (t \sqrt{\lambda}) d \sigma(\lambda),
$$

and $\sigma(\lambda)=\rho_{0}(\lambda)-\frac{2}{\pi} \sqrt{\lambda}$. Setting $x=t$ in (3.2) yields

$$
F(x, x)=2 G_{0}(x, x)+\int_{0}^{x} G_{0}(x, \eta) G_{0}(x, \eta) d \eta,
$$

which means that

$$
F(x, x)-\int_{0}^{x} q_{0}(\eta) d \eta+2 h_{0}=\int_{0}^{x} G_{0}^{2}(x, \eta) d \eta \geq 0 .
$$

It is easy to see that

$$
\begin{aligned}
F(x, x) & =\int_{0}^{\infty} \cos ^{2}(x \sqrt{\lambda}) d \sigma(\lambda) \\
& =\frac{1}{2} \int_{0}^{\infty} \cos (2 x \sqrt{\lambda}) d \sigma(\lambda)+\frac{1}{2} \int_{0}^{\infty} d \sigma(\lambda) .
\end{aligned}
$$

From (3.3) we deduce $F(0,0)=\int_{0}^{\infty} d \sigma(\lambda)$, while (3.4) yields

$$
F(0,0)=-2 h_{0} \text {, }
$$

i.e.

$$
\frac{1}{2} \int_{0}^{\infty} d \sigma(\lambda)=-h_{0}
$$

and thus we can recast (3.4) as

$$
\frac{1}{2} \int_{0}^{\infty} \cos (2 x \sqrt{\lambda}) d \sigma(\lambda)-\int_{0}^{x} q_{0}(\eta) d \eta+h_{0}=\int_{0}^{x} G_{0}^{2}(x, \eta) d \eta \geq 0 .
$$

For the next step we need

Proposition 1. Assume that $q_{0} \in L^{1}(0, \infty)$. Then

$$
\lim _{x \rightarrow \infty} F(x, x)=-h_{0} .
$$


Proof. By (3.5) and (3.6) we see that (3.8) would hold true if we could establish that

$$
\lim _{x \rightarrow \infty} \int_{0}^{\infty} \cos (2 x \sqrt{\lambda}) d \sigma(\lambda)=0 .
$$

The key here is to use the Riemann-Lebesgue theorem. First a classical result from Levitan, [9, Lemma 5.2.2], follows if $q_{0} \in L^{1}(0, \infty)$. Then $\rho_{0}$ is absolutely continuous,

$$
\begin{aligned}
d \rho_{0}(\lambda) & =\frac{1}{\pi} \frac{1}{\sqrt{\lambda}\left(m^{2}(\lambda)+n^{2}(\lambda)\right)} d \lambda \\
& =\frac{2}{\pi} \frac{1}{\left(m^{2}(\lambda)+n^{2}(\lambda)\right)} d \sqrt{\lambda}
\end{aligned}
$$

where

$$
\begin{aligned}
& m(\lambda)=1-\frac{1}{\sqrt{\lambda}} \int_{0}^{\infty} \sin (t \sqrt{\lambda}) q_{0}(t) y_{0}(t, \lambda) d t \quad \text { and } \\
& n(\lambda)=\frac{1}{\sqrt{\lambda}} h-\frac{1}{\sqrt{\lambda}} \int_{0}^{\infty} \cos (t \sqrt{\lambda}) q_{0}(t) y_{0}(t, \lambda) d t .
\end{aligned}
$$

The solution $y_{0}(t, \lambda)$ is bounded. Indeed from the variation of parameters, with $\mu=\sqrt{\lambda}$,

$$
y_{0}(t, \lambda)=\cos (t \mu)+h \frac{\sin (x \mu)}{\mu}+\int_{0}^{x} \frac{1}{\mu} \sin \mu(x-t) q_{0}(t) y_{0}(t, \lambda) d t .
$$

We deduce that $y_{0}(t, \lambda)=\cos (t \mu)+O\left(\frac{1}{\mu}\right)$ as $\mu \rightarrow \infty$, and $\operatorname{sos}_{x \geq 0}\left|y_{0}(x, \lambda)\right|<M$. Since $q_{0} \in L^{1}(0, \infty)$ both functions $n$ and $m$ are well defined, and

$$
\begin{aligned}
m(\lambda) & =1-\frac{1}{2 \mu} \int_{0}^{\infty} \sin (2 \mu t) q_{0}(t) d t+O\left(\frac{1}{\mu^{2}}\right), \\
m^{2}(\lambda)+n^{2}(\lambda) & =1-\frac{1}{\mu} \int_{0}^{\infty} \sin (2 \mu t) q_{0}(t) d t+O\left(\frac{1}{\mu^{2}}\right) .
\end{aligned}
$$

Going back to $\sigma$, we have

$$
\begin{aligned}
d \sigma(\lambda) & =\frac{2}{\pi}\left[\frac{1}{\left(m^{2}(\lambda)+n^{2}(\lambda)\right)}-1\right] d \sqrt{\lambda} \\
& =\frac{2}{\pi}\left[\frac{1}{1-\frac{1}{\mu} \int_{0}^{\infty} \sin (2 \mu t) q(t) d t+O\left(\frac{1}{\mu^{2}}\right)}-1\right] d \mu \\
& =\frac{2}{\pi}\left(\frac{1}{\mu} \int_{0}^{\infty} \sin (2 \mu t) q(t) d t+O\left(\frac{1}{\mu^{2}}\right)\right) d \mu .
\end{aligned}
$$

Thus we have decomposed $d \sigma$ as

$$
d \sigma(\lambda)=S(\mu) d \mu+V(\mu) d \mu,
$$

where $V \in L^{1}(0, \infty)$ and $S(\mu)=\frac{1}{\mu} \int_{0}^{\infty} \sin (2 \mu t) q_{0}(t) d t$. By the Riemann-Lebesgue Theorem we clearly have

$$
\lim _{x \rightarrow \infty} \int_{0}^{\infty} \cos (2 x \mu) V(\mu) d \mu=0 .
$$


The second part can be treated as a composition of the Fourier cosine and sine transforms [4, p. 4]:

$$
\begin{aligned}
\int_{0}^{\infty} \cos (2 x \mu) S(\mu) d \mu & =\int_{0}^{\infty} \cos (2 x \mu) \frac{1}{\mu} \int_{0}^{\infty} \sin (2 \mu t) q_{0}(t) d t d \mu \\
& =\int_{0}^{\infty} \cos (x \eta) \frac{1}{\eta} \int_{0}^{\infty} \sin (\eta t) q_{0}(t) d t d \eta .
\end{aligned}
$$

In order to proceed further we need a result on the Fourier transform.

Lemma 3. Let $q_{0} \in L^{1}(0, \infty)$. Then

$$
\int_{0}^{\infty} \cos (x \eta) \frac{1}{\eta} \int_{0}^{\infty} \sin (\eta t) q_{0}(t) d t d \eta=\frac{\pi}{2} \int_{x}^{\infty} q_{0}(t) d t .
$$

Proof. Since $q_{0} \in L^{1}(0, \infty)$, the $L^{2}$ theory is not applicable. Nevertheless we have

$$
\begin{aligned}
\int_{0}^{\infty} & \cos (x \eta) \frac{1}{\eta} \int_{0}^{\infty} \sin (\eta t) q_{0}(t) d t d \eta \\
= & \lim _{\nu \rightarrow \infty} \int_{0}^{\nu} \cos (x \eta) \frac{1}{\eta} \int_{0}^{\infty} \sin (\eta t) q_{0}(t) d t d \eta \\
= & \lim _{\nu \rightarrow \infty} \int_{0}^{\infty} q_{0}(t) \int_{0}^{\nu} \cos (x \eta) \frac{1}{\eta} \sin (\eta t) d \eta d t \\
= & \frac{1}{2} \lim _{\nu \rightarrow \infty} \int_{0}^{\infty} q_{0}(t) \int_{0}^{\nu} \frac{1}{\eta}(\sin (\eta(x+t)+\sin (\eta(t-x)) d \eta d t
\end{aligned}
$$

Observe that $\int_{0}^{\nu} \frac{1}{\eta} \sin (\eta a) d \eta$ converges boundedly to $\frac{\pi}{2} \operatorname{sign}(a)$ as $\nu \rightarrow \infty$. We can interchange the order of integration and the limit to obtain

$$
\begin{aligned}
\int_{0}^{\infty} & \cos (x \eta) \frac{1}{\eta} \int_{0}^{\infty} \sin (\eta t) q_{0}(t) d t d \eta \\
= & \frac{1}{2} \int_{0}^{\infty} q_{0}(t) \lim _{\nu \rightarrow \infty} \int_{0}^{\nu} \frac{1}{\eta}\left(\operatorname { s i n } \left(\eta(x+t) d \eta+\lim _{\nu \rightarrow \infty} \int_{0}^{\nu} \frac{1}{\eta} \sin (\eta(t-x)) d \eta d t\right.\right. \\
= & \frac{\pi}{4} \int_{0}^{\infty} \operatorname{sign}(x+t) q_{0}(t) d t+\frac{\pi}{4} \int_{0}^{\infty} \operatorname{sign}(t-x) q_{0}(t) d t \\
= & \frac{\pi}{2} \int_{x}^{\infty} q_{0}(t) d t .
\end{aligned}
$$

Thus applying Lemma 3 to (3.14) yields

$$
\lim _{x \rightarrow \infty} \int_{0}^{\infty} \cos (2 x \mu) S(\mu) d \mu=0,
$$

and combining (3.15) and (3.13) yields (3.9), which reduces formula (3.7) to

$$
\int_{0}^{\infty} q_{0}(\eta) d \eta-h_{0} \leq 0
$$


Theorem 2 (Schmincke inequality). Assume that $q_{N} \in L^{1}(0, \infty)$. Then by (3.16), formula (2.8) becomes

$$
\frac{1}{4} \int_{0}^{\infty} q_{N}(\eta) d \eta-\frac{1}{4} h_{N}<\sum_{j=1}^{N} \sqrt{\lambda_{j}}
$$

Proof. Since $N \geq 1$, the norming constant in (2.8), $\alpha_{1}>0$ forces the strict inequality.

Remark 1. We can start from $q_{0}(x)=0, h_{0}=0$, and a pair $\left(\alpha_{1}, \lambda_{1}\right)$ to construct $q_{1} \in L^{1}(0, \infty)$. Since the positive $\alpha_{1}$ can be chosen as small as we like, the constant $\frac{1}{4}$ is sharp.

Proposition 2. Assume that $q \in L^{1}(0, \infty)$ and $\int_{0}^{\infty} q(t) d t>h$. Then the operator

$$
\left\{\begin{array}{l}
-y^{\prime \prime}(x, \lambda)-q(x) y(x, \lambda)=\lambda y \\
y^{\prime}(0, \lambda)-h y(0, \lambda)=0
\end{array}\right.
$$

has at least one negative eigenvalue.

Proof. By $\int_{0}^{\infty}|q(t)| d t<\infty$, the operator

$$
\left\{\begin{array}{l}
-y^{\prime \prime}(x, \lambda)-q(x) y(x, \lambda)=\lambda y \\
y^{\prime}(0, \lambda)-h y(0, \lambda)=0
\end{array}\right.
$$

has a continuous spectrum and $[0, \infty) \subset \sigma$. If we use (3.17), then we obtain that $0<\sum_{j=1}^{N} \sqrt{\lambda_{j}}$ and thus the existence of at least one negative eigenvalue.

Remark 2. A similar result has been proved for the Schrödinger operator on the whole real line in [13, where the potential $q$ is required to be continuous on $\mathbb{R}$ and tends to 0 as $|x| \rightarrow \infty$, and in [14, where the potential $q$ is assumed to satisfy the Faddeev condition $(1+|x|) q(x) \in L^{1}(\mathbb{R})$.

\section{Product of eigenvalues}

In this section we assume additionally that the Faddeev condition holds:

$$
\int_{0}^{\infty}(1+x)|q(x)| d x<\infty .
$$

From (2.2) we already have

$$
\int_{0}^{t} q_{k+1}(\eta) d \eta=\int_{0}^{t} q_{k}(\eta) d \eta-2 \alpha_{k+1}+2 \frac{\alpha_{k+1} y_{k}^{2}\left(t,-\lambda_{k+1}\right)}{1+\alpha_{k+1} \int_{0}^{t} y_{k}^{2}\left(s,-\lambda_{k+1}\right) d s} .
$$


As $y_{k}\left(x,-\lambda_{k+1}\right) \sim e^{x \sqrt{\lambda_{k+1}}}$, another integration leads to

$$
\begin{aligned}
& \int_{0}^{x} \int_{0}^{t}\left(q_{k+1}(\eta)-q_{k}(\eta)\right) d \eta d t \\
& \quad=-2 \alpha_{k+1} x+2 \int_{0}^{x} \frac{\alpha_{k+1} y_{k}^{2}\left(t,-\lambda_{k+1}\right)}{1+\alpha_{k+1} \int_{0}^{t} y_{k}^{2}\left(s,-\lambda_{k+1}\right) d s} d t \\
& \quad=-2 \alpha_{k+1} x+2 \int_{0}^{x} \frac{d}{d t} \ln \left(1+\alpha_{k+1} \int_{0}^{t} y_{k}^{2}\left(s,-\lambda_{k+1}\right) d s\right) d t \\
& \quad=-2 \alpha_{k+1} x+2 \ln \left(1+\alpha_{k+1} \int_{0}^{x} y_{k}^{2}\left(s,-\lambda_{k+1}\right) d s\right) \\
& =-2 \alpha_{k+1} x+2 \ln \left(\alpha_{k+1} \int_{0}^{x} y_{k}^{2}\left(s,-\lambda_{k+1}\right) d s\right)+o(1) \\
& \quad=-2 \alpha_{k+1} x+2 \ln \left(\alpha_{k+1} \frac{e^{2 \sqrt{\lambda_{k+1}} x}}{2 \sqrt{\lambda_{k+1}}}\right)+o(1) \\
& =\left(4 \sqrt{\lambda_{k+1}}-2 \alpha_{k+1}\right) x-2 \ln \left(\frac{2 \sqrt{\lambda_{k+1}}}{\alpha_{k+1}}\right)+o(1) .
\end{aligned}
$$

Recasting (2.7) as

$$
4 \sqrt{\lambda_{k+1}}-2 \alpha_{k+1}=\int_{0}^{\infty}\left(q_{k+1}(\eta)-q_{k}(\eta)\right) d \eta
$$

simplifies the previous integral:

$$
\begin{aligned}
\int_{0}^{x} & (x-\eta)\left(q_{k+1}(\eta)-q_{k}(\eta)\right) d \eta \\
\quad= & x \int_{0}^{\infty}\left(q_{k+1}(\eta)-q_{k}(\eta)\right) d \eta-2 \ln \left(\frac{2 \sqrt{\lambda_{k+1}}}{\alpha_{k+1}}\right)+o(1), \\
2 \ln \left(\frac{2 \sqrt{\lambda_{k+1}}}{\alpha_{k+1}}\right) & \\
= & \int_{0}^{x} \eta\left(q_{k+1}(\eta)-q_{k}(\eta)\right) d \eta+x \int_{x}^{\infty}\left(q_{k+1}(\eta)-q_{k}(\eta)\right) d \eta+o(1) .
\end{aligned}
$$

If $(1+x) q_{N}(x) \in L^{1}(0, \infty)$, then $(1+x) q_{k}(x) \in L^{1}(0, \infty)$ for any $k=0,1, \cdots, N$, and

$$
x \int_{x}^{\infty}\left|q_{k+1}(\eta)-q_{k}(\eta)\right| d \eta \leq \int_{x}^{\infty} \eta\left|q_{k+1}(\eta)-q_{k}(\eta)\right| d \eta=o(1) .
$$

Taking the limit as $x \rightarrow \infty$ yields

$$
\int_{0}^{\infty} \eta\left(q_{k+1}(\eta)-q_{k}(\eta)\right) d \eta=2 \ln \left(\frac{2 \sqrt{\lambda_{k+1}}}{\alpha_{k+1}}\right) .
$$

Now adding more eigenvalues leads to

Proposition 3. Assume that $(1+x) q_{N}(x) \in L^{1}(0, \infty)$. Then

$$
\int_{0}^{\infty} x\left(q_{N}(x)-q_{0}(x)\right) d x=\ln \left(\frac{4 \prod_{j=1}^{N} \lambda_{j}}{\prod_{j=1}^{N} \alpha_{j}^{2}}\right) .
$$




\section{ACKNOWLEDGMENT}

The first author thanks the American Institute of Mathematics for its support during the meeting on low eigenvalues of the Schrödinger operator organized in May 2006.

\section{REFERENCES}

[1] Benguria, R; and Loss, M.; A simple proof of a theorem of Laptev and Weidl. Math. Res. Lett. 7 (2000), no. 2-3, 195-203. MR.1764316 (2001c:81042)

[2] Benguria, R; and Loss, M.; Connection between the Lieb-Thirring conjecture for Schrödinger operators and an isoperimetric problem for ovals on the plane. Partial Differential Equations and Inverse Problems, 53-61, Contemp. Math., 362, Amer. Math. Soc., Providence, RI, 2004. MR2091490 (2005f:81057)

[3] Boumenir, A; A comparison theorem for selfadjoint operators. Proc. Amer. Math. Soc. 111 (1991), 161-175. MR1021896 (91i:47035)

[4] Erdélyi, A.; Magnus, W.; Oberhettinger, F.; and Tricomi, F. G.; Tables of Integral Transforms. Vol. I. Based, in part, on notes left by Harry Bateman. McGraw-Hill Book Company, Inc., New York-Toronto-London, 1954. MR0061695 (15:868a)

[5] Hundertmark, D.; Lieb, E.H.; and Thomas, L.E.; A sharp bound for an eigenvalue moment of the one-dimensional Schrödinger operator. Adv. Theor. Math. Phys. 2 (1998), 719-731. MR:1663336 (2000c:81062)

[6] Gesztesy, F.; and Teschl, G.; On the double commutation method. Proc. Amer. Math. Soc. 124 (1996), no. 6, 1831-1840. MR1322925 (96h:34171)

[7] Gesztesy, F.; A complete spectral characterization of the double commutation method. J. Funct. Anal. 117 (1993), no. 2, 401-446. MR.1244942 (94m:47093)

[8] Laptev, A.; and Weidl, T.; Sharp Lieb-Thirring inequalities in high dimensions, Acta Mathematica 184 (2000), 87-111. MR.1756570 (2001c:35173)

[9] Levitan, B.M.; Expansion in Characteristic Functions of Differential Equations of the Second Order (Russian). Gosudarstv. Izdat. Tehn.-Teor. Lit., Moscow-Leningrad, 1950. MR0036918 $(12: 183 \mathrm{e})$

[10] Levitan, B.M.; Inverse Sturm-Liouville Problems. VSP, Zeist, 1987. MR933088 (89b:34001)

[11] Lieb, E.; and Thirring, W.; Inequalities for the moments of the eigenvalues of the Schrödinger Hamiltonian and their relation to Sobolev inequalities. Studies in Mathematical Physics, Essays in Honor of Valentine Bargmann. Eds: Lieb E., Simon B., Wightman A., Princeton University Press, Princeton, NJ, 1976.

[12] Nămark, M.A.; Linear Differential Operators. Part II: Linear Differential Operators in Hilbert Space, Ungar Publishing Co., New York, 1968. MR0262880(41:7485)

[13] Schmincke, U.W.; On Schrödinger's factorization method for Sturm-Liouville operators. Proc. Roy. Soc. Edinburgh Sect. A 80 (1978), 67-84. MR529570 (80f:34025)

[14] Weidl, T.; On the Lieb-Thirring constants $L_{\gamma, 1}$ for $\gamma \geq 1 / 2$. Commun. Math. Phys. 178 (1996), 135-146. MR1387945 (97c:81039)

[15] Zakharov, V.E.; and Faddeev, L.D.; Korteweg-de Vries equation: A completely integrable Hamiltonian system. Funct. Anal. Appl. 5 (1971), 280-287. Translated from the Russian original in Funkcional. Anal. i Prilozen 5 (1971), no. 4, 18-27. MR0303132 (46:2270)

Department of Mathematics, University of West Georgia, Carrollton, Georgia 30118

E-mail address: boumenir@westga.edu

Department of Mathematics, University of West Georgia, Carrollton, Georgia 30118

E-mail address: vu@westga.edu 